\title{
Ethical Responsibilities of Researchers and Participants in the Development of Multimodal Interaction Corpora
}

\author{
Maria Koutsombogera and Carl Vogel \\ School of Computer Science and Statistics \\ Trinity College Dublin \\ Dublin, Ireland \\ $\{$ koutsomm,vogel\}@scss.tcd.ie
}

\begin{abstract}
Research on modelling multimodal behavior often requires the development of corpora of human-human or humanmachine interactions. The design and implementation of such corpora should attend to ethical principles throughout the overall data management pipeline, from data collection and storage to data processing. This paper describes the methodology we followed to manage the ethical issues that arise for data collection of multimodal interactions, namely the involvement of human participants and the protection of their personal data. We address ethical issues also by linking them to the aspect of the ethical responsibility from the perspectives of both researchers and participants. These topics are addressed based on the experience and observations gathered during the implementation of the MULTISIMO project as a case study.
\end{abstract}

Keywords: multimodal corpus, research ethics, data protection, privacy, ethical responsibility

\section{INTRODUCTION}

Developing multimodal corpora is one of the most important tasks in interaction and behavioural modelling, since informed models are created on the basis of the knowledge reflected in the actual data. Considering the fact that the quality of the models depends on the data-set quality, there is a need to design and implement multimodal, as naturalistic as possible, data collection involving humans engaged in interaction tasks. This raises major ethical considerations related to the privacy of participants and the anonymity of individuals who contribute to data. Thus, a concern in data collection design is that human participants are provided with all the information they need, in the clearest possible way, including the implications of their participation in experimentation, so that they can independently take a decision to participate or not.

It has been stressed that as the technology progresses, new ethical challenges appear, and traditional ethical theories have been questioned in view of new, flexible ethical frameworks reflecting the alignment with technological evolution [1]. As part of his Information Ethics Theory, Floridi also introduces the Resources-Product-Target model, a macro-ethical approach considering the life cycle of an information product coming from an information resource that has en effect on the context, the infosphere where it is created [2].

This is the case for emerging interactive systems where great responsibility is required at all stages of the related data management process. This is in line with privacy principles designed to be applied to general ethics standards, which encompasses all stages of data production, from the proactive planning of the ethical processes to data delivery [3].

The implementation of ethical frameworks related to data collection and data processing methodologies has been a research challenge in the fields of natural language processing, human-human, human-machine and human-robot interaction, as well as affective and social computing. Related systems integrate data from humans from various contexts, including verbal and non-verbal features. Several recommendations have been proposed by the community, while addressing issues that raise ethical considerations. Recent work on this topic deals with ethics in affect and emotion data-sets and systems [4], [5], the definition of ethical limits in robot behavior and in the ethical usage of affective robots [6], privacy protection in natural language processing tasks [7], ownership issues in translation resources [8], transparency issues and fairness in machine learning models [9], ethical issues in crowd-sourcing methods [10], to name a few.

In this paper, we discuss ethical issues arising from our experience in developing the MULTISIMO multimodal data collection. In the specific project we planned to acquire data that will enable the understanding of the human social behavior in multiparty interaction, the structural understanding of the interaction flow, and the modelling of the social communication mechanisms to be integrated into intelligent collaborative systems exhibiting natural behavior. In this respect, the observation of human-human interaction through a recorded collection is crucial for our work, since it enables us to improve our understanding of the way the dialogue is structured and interpret the conversational behavior of the participants, hoping to provide insights to the design of dialogue systems and intelligent interfaces that interact with humans.

Furthermore, as supporters of mechanisms that allow the sharing of research results with the academic research community, we believe that making the data visible and discoverable will maximize on the one hand the impact of the dataset itself, ${ }^{1}$ and on the other hand the contribution of the participants in the experiments by making the recorded data

\footnotetext{
${ }^{1}$ Impact, in this sense, may be measured in terms of re-use by us and other researchers pursuing related and unrelated research questions.
} 
reusable. ${ }^{2}$ Therefore, given the research value of such a data collection, our goal was also to design the ethical process in such a way that the data-set would be available to the research community to exploit other facets of it, communicating this possibility to prospective participants.

The psychological perspective (personality, affinities, cognitive capabilities, etc.) of multimodal interaction participants directly influences the nature of infocommunication as well as the the kind of information that is transmitted [11]. In this respect, specification of and adherence to an ethical framework and the methodological issues that this framework entails are inherent part of the research in the CogInfoCom field, specifically related to the types and modes of communications identified in the field of CogInfoCom and the way in which the information is conveyed [12]. The investigation of inter- or intra-cognitive sensor-sharing or sensor-bridging communication modes can be performed through experimentation involving human participants. Moreover, there is ever increasing need for types of data that can be developed from experiments with human participants [13]. Therefore, it seems that an ethical framework is not merely appropriate, but essential.

It is uncontroversial to hold that ethical framework appropriate to current times must take into account the needs and challenges faced by the community (whether construed globally, or locally, and acknowledging that the needs which correspond to some level of community construal may be in conflict with others). It is also standard to suppose that an appropriate ethical framework should address the responsibilities of the researcher in protecting the rights of participants. We argue that suitable ethical framework must also address the rights of researchers ${ }^{3}$ and the responsibilities of participants. In particular, we note the need for a wider and deeper discussion of the ethical responsibilities of research participants.

In the next sections we take a closer look at the ethics procedures that were adopted for the implementation of the data collection (section II) and we discuss challenges experienced during the design and the implementation of the experiments (section III). Finally, we conclude with reflections on improving aspects of the whole process in an attempt to resolve challenging issues.

\section{ETHICAL CONSIDERATIONS IN THE DATA Collection Process}

\section{A. Description of experimental study and related materials}

The aim of the experiments was to collect a corpus of multi-party, task-based interactions, consisting of group sessions. A group of 3 persons, i.e. 2 players and 1 facilitator, was engaged in a collaborative discussion around a game-like task. The task of the players was to collaborate with each other in order to find the 3 most popular answers to each of 3 questions (based on survey questions that were posed to a sample of 100 people), and to order their answers in

\footnotetext{
${ }^{2}$ Impact, in this sense, is measurable in part using the same method as indicated in fn. 1. However, here measures are also attuned to data (like demographic information) about the individuals who participate, in addition to the primary data participants generate.

${ }^{3}$ The right to ask questions is may be thought of as a basic right of researchers. This is distinct from a putative right to have answers to questions.
}

terms of popularity. All sessions were captured using nonintrusive recording equipment, i.e. HD cameras, microphones and Kinect sensors. The data acquisition process took place in the premises of Trinity College Dublin (TCD) and in total 23 sessions were recorded, with an average duration of 10 minutes per session. Survey materials were also employed for the experiments. In order to investigate the effect of personality traits on the flow and the success of the interaction, all participants completed a personality test based on the big five personality traits [14], [15]. Subsequently, the success of the interaction and the task at hand would be correlated with the different personality scores and various combinations of personalities within the groups. Furthermore, after each session, the participants completed a variant of the user experience questionnaire [16], allowing a quick assessment of their impressions from the interaction.

In view of our intention to make this data collection available to the research community, and at the same time protect the participants' rights to privacy and confidentiality, we followed a principled ethical framework process. Two of the most common measures to protect privacy, that is to limit access to personal information and to assure participants' right to control their personal information, are anonymisation and informed consent. Since the data collection would include audio and video from which the participants could be identified, anonymisation was not an option. ${ }^{4}$ Thus, we followed the informed consent practice so that participants would decide whether they'd wish to disclose their personal information to third parties. Thus, the degree of openness of the data eventually depends on the participant's decision, and the level of the consent that obtained through the consent forms.

\section{B. Ethical Board Processes}

Since the specific experiments include recordings of group conversations, with the intention of them being publicly open depending on the consent level of participants, justification and documentation of the methods by which the provisions and research practice guidelines would be met was provided. As is the case with most academic institutions regarding data collections including human participants, we followed the TCD ethical standards and mechanisms that supervise the process. These mechanisms indicate that research should be conducted to the highest ethical standards and each individual researcher is responsible for ensuring good ethical practice, following the good research practice policy [17].

The data collection complied with guidelines of the ethics committee of TCD's School of Computer Science and Statistics (SCSS) and approval policies in place. The board's main task is to consider all steps of the experimental process before any actual data collection occurs, including design principles, participant recruitment and privacy protection. The process followed by the TCD SCSS ethics committee required that the responsible researchers provide the related documentation covering the following aspects: (a) description of project, its processes and ethical considerations, (b) information sheet

\footnotetext{
${ }^{4}$ While participants in the MULTISIMO recordings do not provide their contact details within the recordings, and while the recordings are unlikely to become viral memes or sought by people familiar with the participants, it is possible that someone known to a participant could come across the materials and recognize the participant.
} 
to the participants and (c) consent forms to be signed by the participants and researchers. The ethics board provides constructive feedback on ethical matters and gives approval only when the materials document a satisfactory procedure.

1) Brief description of methods and measurements to be used: The project description included the context of the research and the objectives of the experiments, the targeted number of participants, the place where the experiment would take place, the approximate duration and details about the equipment to be used. The participant recruitment methods, together with details about inclusion and exclusion criteria needed to be further justified. Another prerequisite was to detail the debriefing arrangements. It was documented that a concise debriefing would be performed after each session, aiming to answer all concerns or questions of the participants, mostly ensuring that they felt comfortable throughout the recordings. If requested, further explanations would be given to them regarding the next steps of the research project specifically with regard to the processing of the recordings. Participants would also have access to the scores of their personality tests individually, and will be given pointers to related bibliography in case they wish to read further details.

The most important aspect of the project description is the statement of the ethical considerations raised by the project and the measures with which they would be dealt. This section stresses the voluntary nature of the experiments and the right of participants to withdraw the experiment at any time without penalty. It also lists what is considered personal data. In this respect, personal data refers to the audio and video data collection and the personality tests that participants would take.

In terms of confidentiality, and due to the collection of audio and video data, complete anonymity of the personal data would not be possible. Nevertheless, the project complies with national and European legislation with the aim to safeguard the privacy of individuals, specifically with the primary legislation governing data protection in Ireland, i.e. the Data Protection Act 1988 [18] and the Data Protection (Amendment) Act 2003 [19], which in turn brought the Irish law into line with the EU Data Protection Directive 95/46/EC [20]. Therefore, three levels of consent are identified, i.e. the subjects' details will be fully anonymised and the data collected from the experiment (questionnaires, recorded data) will be used for academic research purposes only (analysis, publication and presentation of resulting data and findings) with complete anonymity, unless participants select otherwise in their consent form, by opt-in, (a) to allow the researchers/research team to use the audio and/or video data in scientific presentations, or (b) to allow the reuse of the data by other qualified researchers, or (c) to allow public use of the data. Personal data would not be used or disclosed in any manner incompatible with these purposes, and in the event where participants do not opt for open access, the specific data-sets/sessions conforms to the standards of anonymity preservation and data retention. Finally, it was made clear that if the level of consent will allow for data sharing, the corpus will be linked to a repository to provide longterm access, yet restricted to some extent, depending on the licensing schemes applied to the data.

2) Information sheet: The information sheet was designed to provide information about the context of the research and the relevance of the experimental study, as well as detail the procedures that are relevant to the participant, including the task description they'll be involved in, its duration, stressing the participants' right to withdraw and to receive debriefing after participation. An important part of the information sheet is that of informing them about anticipated risks or benefits, the decisions they have to take (options in the consent form), as well as long-term decisions, i.e. how to contact the responsible researchers in case they want to withdraw consent after the experiments, but also the provisions taken to protect their privacy and personal information. In this respect, the participants are informed that they will be recorded and observed and that all personal data will be fairly obtained, since they will be aware of the purpose for which the responsible researcher is collecting the data; that the researcher is the only person to whom their personality data will be disclosed, unless they state otherwise in the consent forms; of the occasions and dissemination activities where the researcher will show parts of the video they appear in with their permission; of the right to withdraw at any stage of the process, and of their rights to be debriefed and to have access to their individual data; and, finally, that the research in question is not intended to direct commercial or other exploitation.

3) Consent forms: The consent forms content includes again the background and rationale of the research as well as the experiment procedures and the tasks the participants would be involved in (including duration and compensation). An additional section regarding the publication of the experiments results scientific research conferences and journals is also included. The core section in the consent form are the declarations of the participants, stating their understanding of the experiment processes and acknowledging their rights. Thus, the form checks participants' understanding that there will be audio and video recording during the experiment and that due to the nature of audio and video their identity may be recognised by people who know them. Subsequently, regarding the use of the data collection, three options are presented to the prospective participants, i.e. three levels of consent are defined: (a) use for this specific project (MULTISIMO) and related demonstrations, (b) research or teaching use by other qualified researchers and (c) public use and deposition with an open access repository. The information about the participants (name, contact details, age, etc.) will be kept separately from the data in an encrypted file and identifiers will be used on the data files to refer to the participants. The consent form concludes with a statement of investigator's responsibility and the contact details in case participants want to modify their consent status, subsequently to participating.

After the approval of the ethics committee was granted, the recruitment period started. Participants responding to the announcement were sent an electronic copy of the information sheet and the consent form, so that they would be informed in detail about the goals of the experiments and the related research and have the opportunity to discuss with the lead researcher about any questions they may have before signing the consent form.

\section{ChallengeS}

\section{A. Considerations in designing the consent forms}

At the time of the preparation of the relevant documentation to be submitted to the ethical board we were guided by our 
intention to make the resulting data-set publicly available, provided we would get the participants' consent. Thus, our goal was to show that a participant's wish to privacy is a priority. Our goals needed to be worded through a comprehensible vocabulary in such a way that the study would be explained and that potential future data use would be understood by the participants. To this end, an extensive research of best practice has been conducted regarding conceptual frameworks and procedures reflected on consent forms, as well as for the documentation accompanying resources that are available in open access repositories. We first investigated largely used and acknowledged multimodal corpora whose related consent forms were however rudimentary, since most of these resources we recollected in the early 00's when the open data initiative was not yet widely adopted. Relevant and interesting information was found nevertheless in the practices used by a few academic institutions in Europe, which use consent forms that define 3 layers of consent, i.e. for internal use, for extended research use, and for public use. Moreover, we examined existing open language resource repositories and language resource distribution agencies, which have a well-defined framework for licensing the resources under varying permissions (e.g. allow or not derivatives, allow or not commercial use etc.) or for providing templates of end-user agreements.

\section{B. Understanding the consent forms}

The informed consent forms are a tool that enables participants have control over their personal identification, something which is considered crucial [21], [22]. The use of consent forms has, however, certain limitations regarding the way they are experienced by the participants. Specifically, it has been pointed out that participants "don't always read them, and if they do, they don't always understand them; if they do understand them, they may lack background knowledge, or their choice might be skewed by difficulties in decision making" [23]. To handle this issue, the responsible researchers emailed the consent form templates together with the information sheet as soon as participants expressed interest participating and well before the experiment took place, so that the participants would be aware of the background research and the decision they would need to take. On the day of the experiment and before its start, the researchers invested enough time to explain to the participants the options they needed to make before signing the consent form. The researchers stressed the existence of the clause (option) that their recorded session would be publicly available if they opted for it. This was emphasized to ensure that the participants actually understood the text of the form, as it was attested that more than half of the participants did not read the consent form before the experiment day.

\section{Participants' responses to the ethical procedure}

The process of communicating the goals of the experiments and the role of the participants in them was transparent, as every prospective participant was informed about the ethical considerations that the experiments raise from the very beginning. From the research point of view, this entails two risks. A first risk was losing potential participants while communicating that the experiment would involve audio/video recordings. Among other reasons (e.g. no availability at the date and time of the experiments), one possible explanation would be that they may not realise or misinterpret the privacy protection mechanism foreseen through the consent forms. Actually, a $3 \%$ of the people originally interested in participating in the experiments refused to be involved after they were emailed the details of the experiment (consent form $\&$ information sheet).

A second risk was that participants would not opt for public availability of their data/session. This was somehow expected, since we did not presuppose that all participants would be in favour of data openness. This is a more serious risk, given that most of the time this decision took place right before the experiment. Therefore, if one of the three participants involved in the group session does not opt for openness, this would affect the availability potential of the whole session, since in the group discussion all three members would be viewed and heard. To emphasize, we did not adopt the within-session majority view of how open the session data would be, but the most restrictive view supplied by any individual within the session. There is of course the option of post-processing and anonymization; however, given the goal of the data-set, i.e. to study multimodal behavior, blurring faces or distorting voices is something that was not desired. Therefore, participants' options resulted to the fact that 5 out of the 23 recorded sessions will not be included in the data collection that will be openly accessible, but will be used of course only for the project for which they were initially collected.

In terms of participants' reactions to this specific issue we noticed contrasting opinions: there were participants who understood the value of the data-set and would support that it would be openly available. On the other hand there were participants selecting the internal use only, some of them specifically claiming that their photos or videos should not be used in any dissemination or publication efforts. An interesting fact was that participants did not show any concern regarding the personality test information, which is highly private. This could be explained partly through the provisions already taken in the consent form, where it was stressed that the use of this personality test would be done for the purposes of this experiment only (i.e. to correlate personality traits with behavioral aspects) and that results of this test are not psychological or psychiatric advice of any kind and come with no guarantee of accuracy or fitness for a particular purpose. However, we believe that the main reason for this absence of reaction was that audiovisual identification was perceived as a higher-risk task than textual information provided in the tests.

\section{Ethical Responsibility}

The ethical responsibility as part of being involved in a research experiment has been mostly focused on the researcher's perspective, and not enough concern has been given to participant's perspective. As far as the researchers are concerned, they are responsible for identifying actual and potential ethical issues, reflect upon them and formulate procedures to deal with them. They implement the ethical procedures and update them if necessary, depending on participants' feedback. The lead researchers' responsibility is highlighted also through a statement included in the consent forms, stressing that they have explained the purpose and the details of the study, that they have answered any participants' questions and that the participant has freely given informed consent. They are 
committed to the provisions of data protection act and the College's Policy on Good Research Practice.

Risks to research arising from participant recalcitrance has been discussed in the research methods literature (e.g. [24], [25, pp. 184-189]), and these risks are as important to guard against as well-intentioned participant-related confounds, for example, demand characteristics [26]. The literature addresses, for example, estimating whether research participants participated in earnest [27], [28] or establishing methods to improve response quality [29]. However, we are not aware of prior discussion of the ethical responsibilities of research participants that accompany their ethical rights.

It was attested that the participants exercised their right to control their personal information, despite what most people believe today, i.e. that respecting privacy is almost impractical [30], especially considering the extensive use and exposure on social media platforms. We thus believe that participants possess a methodological responsibility in terms of responding to each step of the experimental process and making informed decisions. We argue however, that at times participants do not fulfill ethical responsibilities towards research. We consider ethically responsible a participant who acknowledges both his/her rights as well as responsibilities, i.e. who invests time in reading the documentation related to an experiment; who contacts the lead researchers in case there are any issues to discuss; who respects the collective efforts required to set up a research experiment; who informs well in advance the lead researchers the intention to participate or not; who thinks deeply in advance of participation about the possibility that some years later permission for the data to remain part of collection might be withdrawn. These issues are additional to the usual presuppositions of earnest and honest engagement with the research materials as presented.

A measure to overcome situations as those described above would be to engage participants in a constructive discussion, explaining issues related to the participants' rights, data collection process, the ethical implications, data sharing considerations, data use, and where potential questions would be answered. However, there are practical problems with this recommendation, since it would be almost impossible to convince the participants to dedicate time for two meetings (for debriefing and for the actual experiment respectively). As regards the decision about whether the data will be open or not, there are higher expectations from participants who are involved in research (e.g. post-doc researchers, research assistants, students) compared to non-researchers. The rationale behind this is that the first category belongs to an ecosystem which is largely supported by public funding and it is more likely that this category of participants is more sensitive to open data research, thus more committed to support openness and re-usability of data-sets from the wider research community. In this respect, further activities to raise awareness should be designed, especially in academic environments and targeting even junior researchers. These activities should stress that open research data has become a common practice in the EU in the recent years and results of publicly funded research are often made publicly available, enabling researchers to collaborate, minimize efforts for creating additional data-sets, build upon previous research and improve the quality of research results. It is also important to stress the potential of the participatory design approach [31], which can inspire research study participants acquire a more responsible role, i.e. to share their concerns and interact with the relevant stakeholders by giving feedback to ethical procedures aspects. Their involvement in several stages of the process will help better define the problems as well as discuss possible solutions.

\section{CONCLUSION}

In this paper we argued that there is a need to follow ethics guidelines when conducting experiments that lead to the collection of multimodal interaction data involving humans to ensure their privacy. We hope that sharing our research experience can assist future researchers in developing an ethical framework suitable to their own research.

Making the data collection publicly available for research purposes poses a challenge that needs to be reflected on at all stages of the ethical process. We demonstrated the above through our experience with experiments carried out within the MULTISIMO project. We drew ethical principles from existing ethical frameworks at a national and EU level and committed to the relevant ethical obligations that were recommended by our institution's ethical board as well as the best practice followed in other academic institutions. The approach we used to address privacy issues was the use of informed consent forms. We then discussed difficulties in identifying the appropriate consent form content that would highlight the possibility of the data being publicly available, while at the same time promoting the participants' right to have control over their data. We attested that what consent forms' content design is currently missing are the practical steps to be taken with regards to the general public use of data-sets. We have also pointed out some inherent limitations of the informed consent process, namely in the way it is perceived by the subjects, who don't always have comprehensive or correct understanding of the ethical considerations.

Furthermore, we emphasized a new dimension of ethical responsibility, that of the participants' perspective. To this end, we strongly support the development of an ethical culture, a community that will discuss and reflect upon ethical issues through an interdisciplinary approach, i.e. including experts from different research fields (data science, sociology psychology, philosophy, etc.) who would exchange views on ethical issues they encounter in their research and develop a critical perspective on that matter. More training of senior and junior researchers in the academic environment on the conduct of ethical research should be pursued. Most importantly, the nonexpert audience, i.e. the usual pool of participants, should not be excluded from this process; instead, it should have access to the ethical notions and the related public discussions. Similar to the participatory design approach, research study participants will influence the design of ethical procedures to meet their needs as they are themselves the intended addressees. In that way, participant involvement in ethics policy discussion is similar to citizen involvement in EU policies and in line with the EU co-creation concept aiming at inclusive and reflective societies. Since there is no one-size-fits-all approach, the profound understanding of the participants' needs and the research needs on the other hand, are important elements in the attempt to update ethical frameworks that fulfill those needs. Encouraging collaboration and involvement of both experts 
and non-experts would achieve shared understanding of the related issues, mutual contribution of efforts and ideas to make effective decisions and to better shape the ethical ecosystem.

\section{ACKNOWLEDGMENT}

The research leading to these results has received funding from the European Union's Horizon 2020 research and innovation programme under the Marie Sklodowska-Curie grant agreement No 701621 (MULTISIMO).

\section{REFERENCES}

[1] L. Floridi, "Information ethics: On the philosophical foundation of computer ethics," Ethics and information technology, vol. 1, no. 1, pp. 33-52, 1999.

[2] - The ethics of information. Oxford University Press, 2013.

[3] A. Cavoukian, "Privacy by design," The Office of the Information and Privacy Commissioner of Ontario, Toronto, Ontario, Canada, Tech. Rep., 2009.

[4] C. Reynolds and R. Picard, "Affective sensors, privacy, and ethical contracts," in CHI 04 Extended Abstracts on Human Factors in Computing Systems. ACM, 2004, pp. 1103-1106.

[5] B. Schuller, J.-G. Ganascia, and L. Devillers, "Multimodal sentiment analysis in the wild: Ethical considerations on data collection, annotation, and exploitation," in Proceedings of Workshop on Ethics In Corpus Collection, Annotation \& Application (ETHI-CA2), LREC, Portoroz, Slovenia, 2016.

[6] L. Bechade, A. Delaborde, G. D. Duplessis, and L. Devillers, "Ethical considerations and feedback from social human-robot interaction with elderly people," in Proceedings of Workshop on Ethics In Corpus Collection, Annotation \& Application (ETHI-CA2), LREC, Portoroz, Slovenia, 2016.

[7] D. Hovy, S. Spruit, M. Mitchell, E. M. Bender, M. Strube, and H. Wallach, Eds., Proceedings of the First ACL Workshop on Ethics in Natural Language Processing. Valencia, Spain: Association for Computational Linguistics, April 2017. [Online]. Available: http://www.aclweb.org/anthology/W/W17/W17-1600

[8] J. Moorkens, D. Lewis, W. Reijers, E. Vanmassenhove, and A. Way, "Translation resources and translator disempowerment," in Proceedings of Workshop on Ethics In Corpus Collection, Annotation \& Application (ETHI-CA2), LREC, Portoroz, Slovenia, 2016.

[9] (2017) Fat-ml: Fairness, accountability, and transparency in machine learning. [Online]. Available: http://www.fatml.org/

[10] K. B. Cohen, K. Fort, G. Adda, S. Zhou, and D. Farri, "Ethical issues in corpus linguistics and annotation: Pay per hit does not affect effective hourly rate for linguistic resource development on amazon mechanical turk," in Proceedings of Workshop on Ethics In Corpus Collection, Annotation \& Application (ETHI-CA2), LREC, Portoroz Slovenia, 2016.

[11] G. Sallai, "Defining infocommunications and related terms," Acta Polytechnica Hungarica, vol. 9, no. 6, pp. 5-15, 2012.

[12] P. Baranyi and A. Csapo, "Definition and synergies of cognitive infocommunications," Acta Polytechnica Hungarica, vol. 9, no. 1, pp. 67-83, 2012.

[13] A. Esposito, A. M. Esposito, and C. Vogel, "Needs and challenges in human computer interaction for processing social emotional information," Pattern Recognition Letters, vol. 66, pp. 41-51, 2015.

[14] O. P. John, E. M. Donahue, and R. L. Kentle, "The big five inventory versions 4a and 54," 1991.

[15] O. P. John, L. P. Naumann, and C. J. Soto, "Paradigm shift to the integrative big five trait taxonomy," Handbook of personality: Theory and research, vol. 3, pp. 114-158, 2008.

[16] B. Laugwitz, T. Held, and M. Schrepp, "Construction and evaluation of a user experience questionnaire," in Symposium of the Austrian HCI and Usability Engineering Group. Springer Berlin Heidelberg, 2008, pp. 63-76.

[17] (2017) Tcd information compliance: Data protection. [Online]. Available: http://www.tcd.ie/info_compliance/data-protection/ $\begin{array}{lrcrl}\text { [18] } & \text { (1988) } & \text { Data } & \text { protection } & \text { act, } \\ \text { ernment } & \text { of } & \text { ireland. } & \text { [Online]. } & \text { gov- } \\ \text { Available: }\end{array}$ http://www.irishstatutebook.ie/eli/1988/act/25/enacted/en/html

[19] (2003) Data protection (amendment) act 2003 , government of ireland. [Online]. Available: http://www.irishstatutebook.ie/eli/2003/act/6/enacted/en/html

[20] (1995) Directive 95/46/ec of the european parliament and of the council of 24 october 1995. [Online]. Available: http://eur-lex.europa.eu/legalcontent/EN/TXT/uri=celex:31995L0046

[21] D. Solove, "Conceptualizing privacy," California Law Review, vol. 90, no. 4, pp. 1087-1155, 2002

[22] H. Nissenbaum, Privacy in Context: Technology, Policy, and the Integrity of Social Life. Stanford, CA, USA: Stanford University Press, 2009.

[23] D. Solove, "Privacy management and the consent dilemma," Harvard Law Review, vol. 126, pp. 1830-1903, 2013.

[24] C. Hewson, D. Laurent, and C. Vogel, "Proper methodologies for psychological and sociological experiments administered via internet," Behavior Research Methods, Instruments, \& Computers, vol. 28, no. 2, pp. 186-191, 1996.

[25] C. Hewson, C. Vogel, and D. Laurent, Internet Research Methods, 2nd Ed. London: SAGE, 2016.

[26] R. Rosenthal, "Biasing effects of experimenters," ETC: A Review of General Semantics, vol. 34, pp. 253-264, 1977.

[27] A. W. Meade and S. B. Craig, "Identifying careless responses in survey data," Psychological Methods, vol. 17, no. 3, pp. 437-455, 2012.

[28] J. L. Huang, P. G. Curran, J. Keeney, E. M. Poposki, and R. P. DeShon, "Detecting and deterring insufficient effort responding to surveys," Journal of Business and Psychology, vol. 27, no. 1, pp. 99-114, 2012.

[29] E. Deutskens, K. de Ruyter, M. Wetzels, and P. Oosterveld, "Response rate and response quality of internet-based surveys: An experimental study," Marketing Letters, vol. 15, no. 1, pp. 21-36, 2004.

[30] D. Solove, "I have got nothing to hide and other misunderstanding of privacy," San Diego Law Review, vol. 44, pp. 745-757, 2007.

[31] E. B.-N. Sanders and P. J. Stappers, "Co-creation and the new landscapes of design," Co-design, vol. 4, no. 1, pp. 5-18, 2008. 